\title{
Mild AKI is associated with mortality of patients who underwent cardiopulmonary bypass surgery
}

\author{
YANLI YANG and JUN MA
}

\author{
Department for Anesthesiology, Beijing Anzhen Hospital, Capital Medical University, Beijing 100029, P.R. China
}

Received December 19, 2018; Accepted November 27, 2019

DOI: $10.3892 /$ etm.2020.9039

\begin{abstract}
Acute kidney injury (AKI) stage I is the most common stage of AKI observed among patients who underwent cardiac surgery with cardiopulmonary bypass (CPB). The relationship between AKI stage I and mortality requires further investigation. Patients aged 18 years or older who underwent cardiac surgery with CPB between July 1, 2013 and May 31, 2014, were reviewed in the present study. Patients were dichotomized into: i) The AKI stage I group, and ii) the non-AKI group. The primary measured characteristic in the present study was the relationship between AKI and mortality. Kaplan-Meier survival analyses were taken to obtain survival curves. A total of 1,846 patients were included in this present study. The mean age was $51.76 \pm 13.56$ years. A total of 1,508 patients did not develop AKI and 338 developed AKI stage I. The mean follow-up period among survivors was $9.95 \pm 3.45$ months. Kaplan-Meier survival analyses showed that patients with AKI stage I were at an increased mortality risk $(\mathrm{P}<0.0001)$. In multivariate Cox regression analysis, AKI stage I remained independently associated with a reduced survival. Using a subgroup analysis, patients with non-recovery AKI (defined as non-recovery AKI if the serum level does not return before surgery) had a higher mortality rate than patients with recovery AKI $(\mathrm{P}<0.0001)$. AKI stage $\mathrm{I}$ is the most common form of AKI and it is independently related to all-cause mortality in patients who underwent cardiovascular surgery with cardiopulmonary bypass.
\end{abstract}

\section{Introduction}

Acute kidney injury (AKI) is a serious postoperative complication of cardiopulmonary bypass (CPB) and may affect up to $50 \%$ of patients (1). AKI after cardiac surgery is the second most common form of AKI that is treated within the intensive care unit (ICU), second only to septic shock related-AKI (2).

Correspondence to: Dr Jun Ma, Department for Anesthesiology, Beijing Anzhen Hospital, Capital Medical University, 2 Anzhen Road, Beijing 100029, P.R. China

E-mail: junma798@163.com

Key words: acute kidney injury, mortality, cardiovascular surgery
AKI after cardiac surgery is associated with increased mortality and morbidity; however, there is a great variation in the AKI severities. AKI which requires continuous renal replacement treatment, only constitutes $\sim 5 \%$ of total patients with AKI (3-5). It has been reported that a small increase in serum creatinine after cardiac surgery is also associated with an increase in the 30-day mortality rate (6). Mild increases of the serum creatinine levels, manifested as AKI stage I, was the main pattern observed among patients who underwent cardiac surgery. The incidence of mild AKI; however, shows a wide variation in the reported literature. It is reported that patients with persistent renal dysfunction have a higher long-term mortality risk compared with patients who display renal function recovery to baseline levels (7). The mechanisms of action behind AKI after cardiac surgery may be related to hemodynamic changes, ischemia/reperfusion injury, inflammatory factors, microembolization and toxins. AKI stage I requires commonly conservative treatment (if CRRT is not required), which is cost-effective, but maintains a high morbidity. If patients receive insufficient attention, a poor prognosis may result in increased mortality compared to other forms of AKI. The effect of AKI stage I on mortality needs further investigation to ensure that suitable clinical treatment is available for postoperative patients of cardiac surgery.

To this end, the objective of the present study was to define the association between AKI stage I and the mortality among patients undergoing cardiovascular surgery with $\mathrm{CPB}$.

\section{Subjects and methods}

Study population. This prospective observational study was conducted at the Department of Anesthesiology of Beijing Anzhen Hospital, Capital Medical University, Beijing, China. The protocol was approved by the Ethics Committee of Beijing Anzhen Hospital, Capital Medical University. Patients or their family members were fully informed of the study details and signed informed consent forms. All of the eligible patients (male: 1,788 and female: 1,080; age, $>18$ years old) were selected from among inpatients who underwent cardiac surgery with CPB between July 1, 2013, and May 31, 2014.

Exclusion criteria. For each patient, information was collected on the demographics, surgical procedure, transfusion and complications from the internal hospital anesthetic database and clinical information system using. The following exclusion 
criteria were used: i) No serum creatinine indicated before the operation; ii) no serum creatinine after the operation; iii) mortality within the course of surgery; iv) cardiac transplantation; v) aortic surgery with kidney arteries involved, or vi) patients with AKI stage II/III.

Definitions. AKI was defined according to the Kidney Disease Improving Global Outcomes criteria (8). Stage I corresponds to serum creatinine levels $>26.5 \mu \mathrm{mol} / 1(0.3 \mathrm{mg} / \mathrm{dl})$ or levels 1.5-1.9 times greater than baseline levels; stage II corresponds to serum creatinine levels 2.0-2.9 times greater than baseline levels; stage III corresponds to serum creatinine levels $>353.6 \mu \mathrm{mol} / 1$ (4.0 mg/dl), or levels greater than 3.0 times higher than baseline levels, or patients who had begun renal replacement therapy. Urine output criteria were not used in the present study. In the present study, patients were dichotomized into non-AKI group and AKI stage I group. Patients with AKI stage I were included in the recovery group if the serum creatinine levels at discharge decreased, otherwise patients were included in the non-recovery group. All-cause mortality was defined as death at a final follow-up (December 17, 2014), which was obtained by telephone consultation. The primary endpoint of this present study was to examine the association of AKI stage I with all-cause mortality. The second endpoint of the present study was to examine 30-day mortality, length of hospital stay after surgery, length of stay in the ICU, mechanical ventilation time and the post-operation morbidity.

Statistical analysis. Data are presented as the mean \pm standard deviation when normally distributed, as median and interquartile range when data are skewed, and as frequencies and percentages for categorical variables. Patient demographics, intraoperative variables and measures of renal function were compared between the non-AKI patients group and the AKI stage I group using Student t-test for normally distributed data, Kruskal-Wallis test or $\chi^{2}$ test when appropriate. Kaplan-Meier survival analysis was used to draw survival curves, which were analyzed using log-rank test. A multivariate Cox proportional hazards survival model was used to estimate hazard ratios (HRs) with 95\% confidence intervals (CIs). Two-sided $\mathrm{P}$ values were used, and $\mathrm{P}<0.05$ was considered to indicate a statistically significant difference.

\section{Results}

Baseline characteristics. A total of 2,868 patients were screened following cardiac surgery with CPB between July 1, 2013, and May 31, 2014. Fig. 1 shows the flowchart of this study, including the included and excluded patients. In total, 1,941 patients were included, with 433 (22.31\%) AKI patients. Of those AKI patients, 338 (78.06\%) were AKI stage I patients (Fig. 2). Finally, 1,846 patients constituted the study population. Of the study patients, the mean age was $51.76 \pm 13.56$ years and $856(46.37 \%)$ were men. Valve surgery accounted for $63.81 \%$ of surgical types, and coronary artery bypass graft $(\mathrm{CABG})+$ valve surgery was the second predominantly used surgical type $(9.15 \%)$. CABG + vascular surgery had the highest incidence of AKI stage I, followed by CABG + valve surgery (Table I). Among the 1,846 patients
2868 Patients undergoing cardiac surgery with cardiopulmonary

256 Aortic surgery with renal arterial involved

2612 Patients

361 Without preoperative creatinine measurement

2251 Patients

303 Without postoperative creatinine measurement

1948 Patients

4 Cardiac transplantation

1944 Patients

3 Died during surgery

1941 Patients

57 AKI stage 2

1884 Patients

38 AKI stage 3

1846 Patients included in this study

Figure 1. Flowchart of included and excluded patients in the present study. AKI, acute kidney injury.

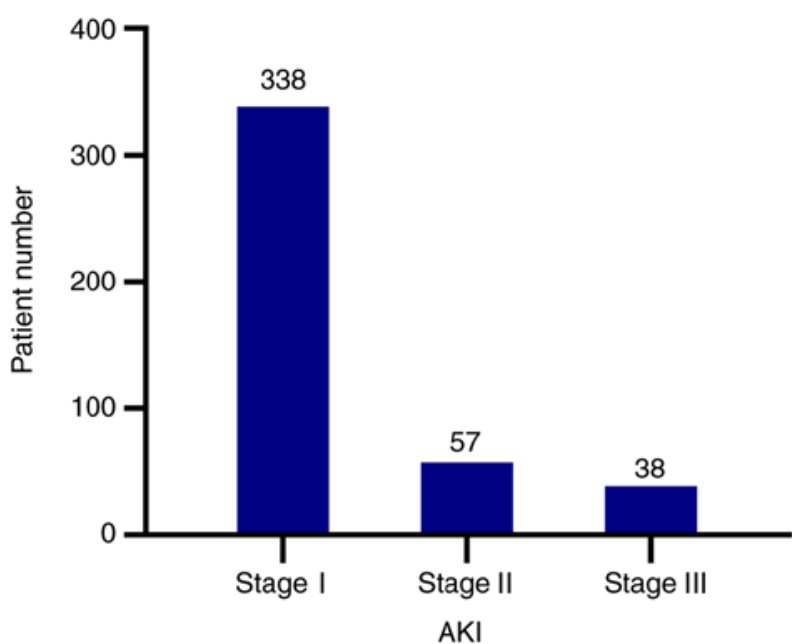

Figure 2. Incidence of the three stages of AKI after cardiac surgery with cardiopulmonary bypass. AKI, acute kidney injury.

included in this study, 1,508 patients did not have AKI and 338 had AKI stage I. There were older patients in AKI stage I group than the non-AKI group. The AKI stage I patients demonstrated a greater proportion of comorbidities of coronary heart disease, hypertension and/or diabetes, compared with the non-AKI group (Table II).

AKI stage I and mortality. Mortality data of all patients were available. The mean follow-up period among survivors was $9.95 \pm 3.45$ months (5.5 days to 15.5 months). Kaplan-Meier survival curves examining the variation between the two 
Table I. Surgery types and patients with AKI stage I.

\begin{tabular}{lcr} 
Surgery & All cases, $n(\%)$ & with AKI stage I, n $(\%)$ \\
\hline Valve surgery & $1178(63.81)$ & $227(19.27)$ \\
CABG & $26(1.41)$ & $5(19.23)$ \\
CABG + valve surgery & $169(9.15)$ & $58(33.58)$ \\
Vascular surgery & $166(8.99)$ & $22(13.25)$ \\
Congenital disease surgery & $173(9.37)$ & $5(2.89)$ \\
Valve + vascular surgery & $31(1.68)$ & $8(25.8)$ \\
CABG + congenital disease surgery & $6(0.33)$ & $2(33.33)$ \\
Cardiac tumor surgery & $48(2.60)$ & $5(1.42)$ \\
Congenital disease + valve surgery & $38(2.06)$ & $0(0.00)$ \\
CABG + vascular surgery & $11(0.59)$ & $6(54.55)$
\end{tabular}

AKI, acute kidney injury; CABG, coronary arterial bypass graft.

Table II. Characteristics in the non-AKI group and the AKI stage I group.

\begin{tabular}{|c|c|c|c|}
\hline Variables & non-AKI group $(\mathrm{n}=1,508)$ & AKI stage I group $(n=338)$ & P-value \\
\hline Sex, male & 733 & 169 & 0.074 \\
\hline Age, year & $50.37 \pm 13.57$ & $57.99 \pm 11.68$ & $<0.0001$ \\
\hline Eject fraction, $\%$ & $60.85 \pm 8.4$ & $58.34 \pm 10.3$ & $<0.0001$ \\
\hline $\mathrm{sCR}$ before surgery, $\mathrm{mmol} / \mathrm{l}$ & $72.57 \pm 18.35$ & $76.33 \pm 40.53$ & $<0.0001$ \\
\hline CPB time, mins & $109.34 \pm 50.04$ & $138.29 \pm 59.74$ & $<0.0001$ \\
\hline Aortic cross-clamp, mins & $75.23 \pm 35.22$ & $92.19 \pm 43.45$ & $<0.0001$ \\
\hline $\mathrm{RBC}$ transfusion, $\mathrm{u}$ & $4.72 \pm 10.86$ & $4.88 \pm 8.1$ & 0.056 \\
\hline Blood plasma transfusion, $\mathrm{ml}$ & $421.10 \pm 483.19$ & $614.23 \pm 713.92$ & $<0.0001$ \\
\hline Fluid balance during surgery, $\mathrm{ml}$ & $675.78 \pm 942.43$ & $870.05 \pm 1015.75$ & 0.001 \\
\hline minimally invasive surgery, $n$ & 62 & 5 & 0.02 \\
\hline Coronary artery disease, $n$ & 136 & 65 & $<0.0001$ \\
\hline Hypertension, $\mathrm{n}$ & 174 & 164 & $<0.0001$ \\
\hline Pulmonary artery hypertension, $\mathrm{n}$ & 230 & 43 & 0.24 \\
\hline $\mathrm{DM}, \mathrm{n}$ & 93 & 133 & 0.001 \\
\hline Peripheral vascular disease, $\mathrm{n}$ & 24 & 17 & 0.236 \\
\hline
\end{tabular}

AKI, acute kidney injury; CPB, cardiopulmonary bypass; DM, diabetes mellitus; RBC, red blood cell; sCR, serum creatinine.

groups are shown in Fig. 3. The data showed that patients with AKI stage I were at a higher risk of mortality compared with non-AKI patients $(\mathrm{P}<0.0001)$.

A univariate Cox analysis was performed first (Table III). All the meaningful factors which were significantly associated with AKI stage I were all included in the multivariate Cox analysis. The following factors were included; Age, blood plasma transfusion, fluid balance during surgery, coronary artery disease, diabetes mellitus, and mechanical ventilation time. In multivariate Cox regression analysis, AKI stage I remained independently associated with reduced survival [(HR, 2.412; 95\% CI, 1.40 to 4.15; P =0.001)(Table IV)]. Patients with AKI stage I had a higher 30-day mortality rate than patients without AKI (3.25 vs. $0.025 \%, \mathrm{P}<0.05$; Table V). In a subgroup Kaplan-Meier survival curve analysis, patients

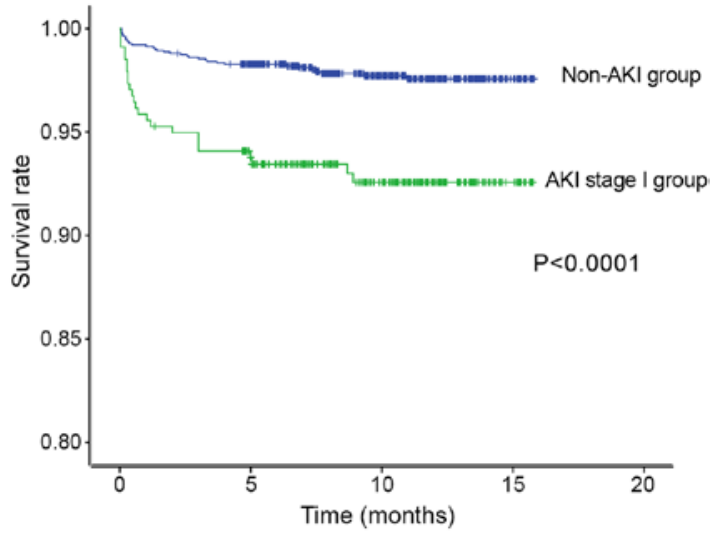

Figure 3. Kaplan-Meier survival analysis of the AKI stage I group and the non-AKI group $(\log$-rank test, $\mathrm{P}<0.0001)$ 
Table III. Factors associated with mortality in univariate analysis.

\begin{tabular}{llr}
\hline Variables & Hazard ratio (95\% CI) & P-value \\
\hline Age & $1.253(1.121-1.405)$ & $<0.00$ \\
Body mass index & $1.110(1.067-1.157)$ & $<0.001$ \\
Hypertension & $1.560(1.203-2.076)$ & 0.002 \\
Chronic kidney disease & $5.70(11.199-8.062)$ & 0.031 \\
Coronary artery disease & $1.903(1.071-3.385)$ & 0.021 \\
Pulmonary artery hypertension & $1.116(1.027-1.210)$ & 0.013 \\
Peripheral vascular disease & $0.983(0.971-1.017)$ & 0.041 \\
sCR before surgery, mmol/l & $3.267(1.932-5.383)$ & $<0.001$ \\
Peripheral vascular disease & $1.050(0.927-1.032)$ & 0.394 \\
Ejection fraction, \% & $0.986(0.972-1.000)$ & 0.053 \\
RBC transfusion & $1.103(0.999-1.050)$ & 0.057 \\
AKI stage I & $2.432(1.41-4.19)$ & 0.001 \\
Blood plasma transfusion, ml & $1.127(1.022-1.226)$ & 0.015 \\
Fluid balance during surgery, ml & $0837(0.671-1.224)$ & 0.542 \\
MAP intraoperation, mmHg & $0.983(0.973-0.982)$ & 0.001 \\
CPB & $1.003(1.001-1.006)$ & 0.033 \\
DM & $1.040(1.02-1.06)$ & 0.008 \\
MV time, day & $1.014(1.009-1.020)$ & $<0.001$
\end{tabular}

AKI, acute kidney injury; CPB, cardiopulmonary bypass; CI, confidence interval; DM, diabetes mellitus; MAP, mean arterial pressure; MV, mechanical ventilation; $\mathrm{RBC}$, red blood cell; sCR, serum creatinine.

Table IV. Factors associated with mortality in multivariate analysis.

\begin{tabular}{lll}
\hline Variables & Hazard ratio $(95 \% \mathrm{CI})$ & P-value \\
\hline AKI stage I & $2.432(1.41-4.19)$ & 0.001 \\
CPB & $1.003(1.001-1.006)$ & 0.033 \\
DM & $1.040(1.02-1.06)$ & 0.008
\end{tabular}

AKI, acute kidney injury; CPB, cardiopulmonary bypass; DM, diabetes mellitus.

with non-recovery AKI had a higher mortality rate than patients with recovery AKI (log-rank test, $\mathrm{P}<0.0001$; Fig. 4). Mechanical ventilation time was longer in the AKI stage I group than that in the non-AKI group $(\mathrm{P}<0.0001)$. Hospital stay after surgery non-AKI group were also longer compared with the non-AKI group (Table V).

\section{Discussion}

In the present study, it was observed that AKI was a common complication of cardiac surgery, and AKI stage I made up to $78 \%$ of all AKI cases. Patients with AKI stage I had a higher 30-day mortality rate compared to patients without AKI. Furthermore, AKI stage I was an independent determinant for all-cause long-term mortality among patients who underwent cardiac surgery. Additionally, patients with AKI stage I had a longer length of hospital stay and required longer treatment

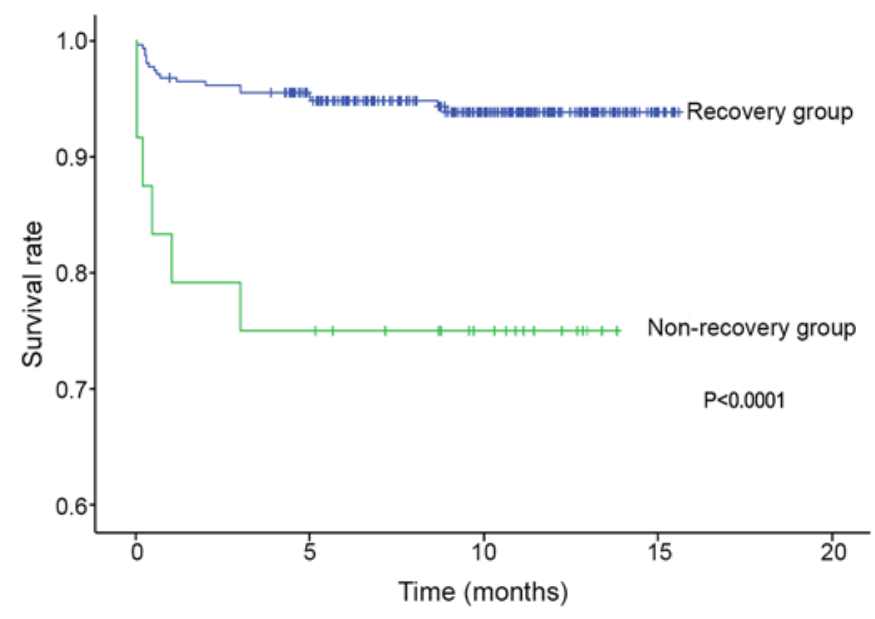

Figure 4. Kaplan-Meier Survival Analysis of recovery-AKI stage I group and non-recovery AKI group (log-rank test, $\mathrm{P}<0.0001)$.

periods than non-AKI patients. Finally, the present study showed that patients with recovery AKI stage I had a better prognosis than those with non-recovery AKI.

The present investigation observed that the main stage of AKI after cardiac surgery with CPB is AKI stage I. AKI is a frequent postoperative clinical complication for patients, especially during cardiac surgery. AKI may affect up to $30 \%$ of patients following cardiac surgery (9). The present study showed that $22.31 \%$ of patients who underwent cardiac surgery developed AKI. The difference between the previously published incidence of AKI and the observation in the present study cannot exclude the improvement of recognition 
Table V. Postoperative characteristics in the non-AKI group and the AKI stage I group.

\begin{tabular}{lccr}
\hline Variables & non-AKI group & AKI stage I group & P-value \\
\hline ICU stay, h & $21.92 \pm 28.88$ & $40.54 \pm 66.45$ & $<0.0001$ \\
Mechanical ventilation time, hours & $21.28 \pm 27.78$ & $38.85 \pm 64.88$ & $<0.0001$ \\
Hospital stay after surgery, days & $8.37 \pm 4.88$ & $10.5 \pm 6.85$ & $<0.0001$ \\
Respiratory dysfunction, $\mathrm{n}$ & 155 & 106 & $<0.0001$ \\
Cardiac events, $\mathrm{n}$ & 9 & 15 & $<0.0001$ \\
30-day mortality, $\mathrm{n}$ & 6 & 11 & $<0.0001$ \\
\hline
\end{tabular}

AKI, acute kidney injury; ICU, intensive care unit.

and treatment in recent years. However, the severity of AKI is vastly different between postoperative AKI and septic AKI. In septic patients, AKI stage III was previously reported to be the main stage (10). In the current investigation, stage I AKI, was the most common stage. It has been previously reported that the incidence of stage I AKI was more than $70 \%$ in patients who underwent cardiac surgery procedures with $\mathrm{CPB}$, which was in agreement with the results of the present study (11). It has been shown that, in patients who had undergone coronary artery bypass grafting, 50\% AKI cases were at stage I (12).

In the present study, patients with AKI stage I had more than a two-fold increase in long-term mortality during a mean follow-up of approximately 10 months, compared with non-AKI patients. In a recent study, Liotta et al (12) reported the AKI stage I patients exhibited a 1.33 increased risk of mortality after 6 years. The present study also found that even minimal increases in the postoperative serum creatinine levels of $<0.3 \mathrm{mg} / \mathrm{dl}$ (26 mmol/l) were related to a significant increase in mortality in patients who had undergone coronary artery bypass graft surgery (11). It was further observed that patients with AKI stage I had a two-fold higher 30-day mortality rate when compared to patients without AKI using propensity matching scores (10).

The present study showed also that patients with stage I AKI were prone to worse postoperative condition than non-AKI patients. ICU stay, mechanical ventilation time and hospital stay were significantly higher longer among patients with AKI stage I than patients without AKI. There were more cases of respiratory dysfunction and adverse cardiac events in patients with AKI stage I compared with patients without AKI. Previous studies also reported similar results (13-15). Furthermore, the present study showed that patients with recovery AKI had a better long-term outcome than those with non-recovery AKI. It has also been reported that the risk of late death (after discharge from hospital) was related to the recovery of AKI and the remaining renal function at discharge (16). Recovery from AKI and the correlation with the long-term outcome of the patient are important issues, which have now recognized as being of key importance (17). Determining the mechanisms by which AKI is associated with mortality is critical, as this may gain a greater understanding of how to provide more effective preventative interventions. However, the occurrence of AKI stage I is likely to be a strong indicator of a poor physiological condition for postoperative patients, as well as having a direct causative influence on mortality. Therefore, it is difficult to determine the modifiable, causative influence of AKI on risk of death. The results of the present study are useful for addressing this issue, as recovery stage from AKI stage I was also related to mortality. The results highlight the significance of prompting treatment for AKI stage I.

The mechanisms underlying the role of AKI stage I in the postoperative course are unclear. Possible pathophysiological mechanisms linking AKI stage I and its clinical effects may include the activation of inflammatory pathways and fluid resuscitation. Activation of inflammatory pathways is another important mechanism behind the development of AKI (18). However, the degree of inflammation may vary from that observed in septic AKI. The level of inflammation may partially explain the difference in the AKI stage between cardiac surgery patients and septic patients (4). Inadequate circulation is common in AKI patients; however, volume overload is a more frequent side-effect of AKI currently (18). This could in turn require patients to extend the time of mechanical ventilation, ICU stay and hospital stay.

It is important to pay more attention to AKI stage I among patients who have undergone cardiac surgery with CPB. Compared with the more severe levels of AKI, there are more patients who develop AKI stage I. In addition, the treatment of AKI stage I may be less invasive and more cost-effective than treating more sever levels of AKI. Therefore, more meticulous diagnosis and treatment might be needed for patients with AKI stage I to ensure a better prognosis.

In conclusion, the present study showed that the AKI stage I was the predominant form of AKI, making up $78 \%$ of all AKI cases. AKI stage I was an independent determinant of all-cause long-term mortality among cardiac surgery patients. In addition, the present study showed that patients with recovery AKI stage I had a better prognosis than those with non-recovery AKI. As there are more patients who develop AKI stage I compared with the other stages, AKI stage I was indicated to lead to as many late deaths associated with AKI compared with patients who develop the more severe stages of AKI. As such, a more meticulous treatment protocol is needed for patients with AKI stage I.

\section{Acknowledgements}

Not applicable. 


\section{Funding}

The present study was funded by grants received from Beijing Municipal Natural Science Foundation (grant no. 7192052); the National Key R\&D Program of China: Perioperative evaluation of acute kidney injury and protection (grant no. 018YFC2001900); and Beijing Municipal Administration of Hospitals Clinical Medicine Development of Special Funding Support (grant no. ZYLX201810).

\section{Availability of data and materials}

The datasets used and/or analyzed during the present study are available from the corresponding author on reasonable request

\section{Authors' contributions}

YYL and JM led the conception and design of this study. YYL and JM were responsible for the data collection and analysis. YYL were in charge of drafting the manuscript. YYL made revision from critical perspective for important intel $\neg$ lectual content. The final version was read and approved by all the authors

\section{Ethics approval and consent to participate}

The protocol was approved by the Ethics Committee of Beijing Anzhen Hospital, Capital Medical University. Patients or their family members were fully informed of the study details and provided their written informed consent.

\section{Patient consent for publication}

Not applicable.

\section{Competing interests}

The authors declare that they have no competing interests.

\section{References}

1. Meersch M, Schmidt C, Hoffmeier A, Van Aken H, Wempe C, Gerss J and Zarbock A: Prevention of cardiac surgery-associated AKI by implementing the KDIGO guidelines in high risk patients identified by biomarkers: The PrevAKI randomized controlled trial. Intensive Care Med 43: 1551-1561, 2017.

2. Uchino S, Kellum JA, Bellomo R, Doig GS, Morimatsu H, Morgera S, Schetz M, Tan I, Bouman C, Macedo E, et al Beginning and ending supportive therapy for the kidney (BEST Kidney) investigators: Acute renal failure in critically ill patients: A multinational, multicenter study. JAMA 294: 813-818, 2005.
3. Ranucci M, Aloisio T, Cazzaniga A, Di Dedda U, Gallazzi C and Pistuddi V: Validation of renal-risk models for the prediction of non-renal replacement therapy cardiac surgery-associated acute kidney injury. Int J Cardiol 272: 49-53, 2018.

4. Mehta RH, Grab JD, O'Brien SM, Bridges CR, Gammie JS, Haan CK, Ferguson TB and Peterson ED; Society of Thoracic Surgeons National Cardiac Surgery Database Investigators: Bedside tool for predicting the risk of postoperative dialysis in patients undergoing cardiac surgery. Circulation 114: 2208-2216, quiz 2208, 2006.

5. Thakar CV, Arrigain S, Worley S, Yared JP and Paganini EP: A clinical score to predict acute renal failure after cardiac surgery. J Am Soc Nephrol 16: 162-168, 2005.

6. Lassnigg A, Schmidlin D, Mouhieddine M, Bachmann LM, Druml W, Bauer P and Hiesmayr M: Minimal changes of serum creatinine predict prognosis in patients after cardiothoracic surgery: A prospective cohort study. J Am Soc Nephrol 15: 1597-1605, 2004.

7. Corredor C, Thomson R and Al-Subaie N: Long-term consequences of acute kidney injury after cardiac surgery: A systematic review and meta-analysis. J Cardiothorac Vasc Anesth 30: 69-75, 2016.

8. Kidney International: KDIGO Clinical Practice Guideline for Acute Kidney Injury. Kidney Int Suppl 2: 1-138, 2012.

9. Thanavaro J, Taylor J, Vitt L and Guignon MS: Predictors and outcomes of acute kidney injury after cardiac surgery. Nephrol Nurs J 46: 31-40, 2019.

10. Kellum JA, Wen X, de Caestecker MP and Hukriede NA: Sepsis-associated acute kidney injury: A problem deserving of new solutions. Nephron 143: 174-178, 2019.

11. Elmistekawy E, McDonald B, Hudson C, Ruel M, Mesana T, Chan V and Boodhwani M: Clinical impact of mild acute kidney injury after cardiac surgery. Ann Thorac Surg 98: 815-822, 2014.

12. Liotta M, Olsson D, Sartipy U and Holzmann MJ: Minimal changes in postoperative creatinine values and early and late mortality and cardiovascular events after coronary artery bypass grafting. Am J Cardiol 113: 70-75, 2014.

13. Hobson CE, Yavas S, Segal MS, Schold JD, Tribble CG, Layon AJ and Bihorac A: Acute kidney injury is associated with increased long-term mortality after cardiothoracic surgery. Circulation 119: 2444-2453, 2009.

14. Gangadharan S, Sundaram KR, Vasudevan S, Ananthakrishnan B, Balachandran R, Cherian A, Varma PK, Gracia LB, Murukan K, Madaiker A, et al: Predictors of acute kidney injury in patients undergoing adult cardiac surgery. Ann Card Anaesth 21: 448-454, 2018.

15. Nadim MK, Forni LG, Bihorac A, et al: Cardiac and Vascular Surgery-Associated Acute Kidney Injury: The 20th international Consensus Conference of the ADQI (Acute Disease Quality Initiative) Group. J Am Heart Assoc 7(11): e008834, 2018.

16. Engoren M, Habib RH, Arslanian-Engoren C, Kheterpal S and Schwann TA: The effect of acute kidney injury and discharge creatinine level on mortality following cardiac surgery. Crit Care Med 42: 2069-2074, 2014

17. Prowle JR and Kirwan CJ: Acute kidney injury after cardiac surgery: The injury that keeps on hurting? Crit Care Med 42: 2142-2143, 2014.

18. Song N, Thaiss F and Guo L: NFxB and kidney injury. Front Immunol 10: 815, 2019.

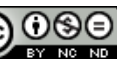

This work is licensed under a Creative Commons Attribution-NonCommercial-NoDerivatives 4.0 International (CC BY-NC-ND 4.0) License. 